\section{On Coordinate-Free Rotation Decomposition: Euler Angles about Arbitrary Axes}

\author{
Giulia Piovan and Francesco Bullo
}

\begin{abstract}
This paper focuses on Euler angles and on the decomposition of rotations. We consider arbitrary rotation axes that are not necessarily mutually orthogonal; we characterize the set of rotation matrices that admit Euler angles about arbitrary rotation axes; and we provide a single set of Euler angles formulas that applies to any selection of rotation axes. The results are presented and derived in a coordinate-free setting, where no reference frames are required and no components of any array or matrix is manipulated.
\end{abstract}

\section{INTRODUCTION}

Rotations matrices play a key role in the field of kinematics and Euler angles are a powerful approach to the decomposition and parametrization of rotation matrices. Accordingly, rotation matrices and Euler angles are standard topics in robotics education, e.g., see the established textbooks [1], [2], [3]. Euler angles are studied in classical and geometric mechanics [4], [5], [6] and are an example of "exponential coordinates of the second kind" in differential geometry [7]. A matrix exponential formalism for rotations is described in [8] and an early reference on the role of matrix exponential in robotics is [9]. An insightful and geometric treatment of rotation matrices in the context of manipulation is given in [10]. Application of Euler angles to flight control are discussed in [11] and to computer vision in [12]. The problem of Euler angles about non-orthogonal axes was first considered by Daveport [13], who proved the conditions for the existence of a solution if the first two and the last two axes are mutually perpendicular. The generalized Euler angles have been successively studied in [14], [15], where the problem was tackled in a coordinate-dependent way.

This paper considers the following problem: can a rotation be equivalent to the composition of three subsequent rotations about given axes? If so, what are the three angles establishing this decomposition? When the three rotation axes are selected from an orthonormal basis, the three angles are the well-known Euler angles. (In other words, decomposing the rotation matrix is equivalent to computing Euler angles.) We are interested in the general case where the three axes are arbitrary and not necessarily mutually orthogonal. Additionally, we tackle this rotation decomposition problem in a coordinate-free way. By coordinate free, we mean that all statements and proofs require neither a reference frame nor the manipulation of the components of any array or matrix.

Our interest for the coordinate-free study of Euler angles about arbitrary axes has multiple motivations. First, we believe that the decomposition of rotations is a fundamental essential problem and that our general discussion has a tutorial value in graduate courses on robotics. Our interest for coordinate-free characterizations and Euler angles formulas is part of an ambitious plan to provide a geometric treatment to the entire field of kinematics. Moreover, Euler angles about arbitrary axes have been recently applied to a number of distinct disciplines. The early work [14] is motivated by applications in multi-body mechanics. The spacecraft and underwater vehicles reorientation problem via a minimal number of rotational maneuvers is discussed in [15] and [16]. Attitude estimation problems involving non-orthogonal Euler angles are discussed in [17]. Finally, the recent

This work was supported in part by NSF Award CNS-0834446. The authors thank Dr. Brian D. O. Anderson for an early insightful discussion.

Giulia Piovan and Francesco Bullo are with the Center for Control, Dynamical Systems and Computation, University of California at Santa Barbara, Santa Barbara, CA 93106, \{giulia, bullo\}eengineering.ucsb.edu work [18] about sensor networks provides necessary and sufficient for the orientation localization problem with only bearing angle information.

We articulate the contributions of this paper as follows. First, in Section II, we characterize three basic problems involving rotation matrices; while the results are simple, they are elegant and of possible general interest. Second, as main result in Section III, we establish necessary and sufficient coordinate-free conditions for the existence of Euler angles about the given arbitrary axes. In other words, as a function of the rotation axes, we characterize the set of rotations that admit the decomposition into subsequent rotations. Remarkably, not all matrices are decomposable when the rotation axes are not mutually orthogonal. Third, if the Euler angles exist, then we provide explicit formulas for their computation. Remarkably, our single set of formulas applies to any possible choice of rotation axes; this statement contrasts with the usual need to handle separately distinct choices of rotation axes. Recall that 12 distinct choices of rotation axes triplets are possible and require corresponding inversion formulas. As fourth and final contribution, in Section IV, we verify that our general conditions and formulas are consistent with the classic results: (i) if the second rotation axis is orthogonal to the first and to the third, then the decomposability conditions are satisfied always and Euler angles exist for any rotation matrix; (ii) our formulas are equivalent to the standard ones for the case of $\mathrm{ZYZ}$ Euler angles.

Compared with the treatment in [14] and in [15], our results and proofs are presented in a geometric coordinate-free fashion and are stated in a particularly explicit and concise way.

\section{A. Basic properties of rotation matrices}

As usual, we define the operator $\widehat{\boldsymbol{v}}: \mathbb{R}^{3} \rightarrow \mathbb{R}^{3}$ by $\widehat{\boldsymbol{v}} \boldsymbol{w}=\boldsymbol{v} \times \boldsymbol{w}$, for all $\boldsymbol{v}, \boldsymbol{w} \in \mathbb{R}^{3}$. Given a unit-length vector $\boldsymbol{n} \in \mathbb{R}^{3}$, Rodrigues' formula expresses the rotation matrix about the rotation axis $\boldsymbol{n}$ of an arbitrary angle $\alpha \in[-\pi, \pi[$ as

$$
\exp (\alpha \widehat{\boldsymbol{n}})=I_{3}+\sin (\alpha) \widehat{\boldsymbol{n}}+(1-\cos (\alpha)) \widehat{\boldsymbol{n}}^{2} .
$$

For any unit-length vector $\boldsymbol{n}$ and angle $\alpha$, the rotation axis $\boldsymbol{n}$ is invariant under any rotation about itself:

$$
\boldsymbol{n}^{T} \exp (\alpha \widehat{\boldsymbol{n}})=\boldsymbol{n}^{T}, \quad \text { and } \quad \exp (\alpha \widehat{\boldsymbol{n}}) \boldsymbol{n}=\boldsymbol{n} .
$$

Finally, we recall that, for any $R \in S O(3)$ and $\boldsymbol{v} \in \mathbb{R}^{3}$,

$$
\boldsymbol{R} \exp (\widehat{\boldsymbol{v}})=\exp (\widehat{\boldsymbol{R v}}) \boldsymbol{R} .
$$

\section{B. Basic trigonometric inversion}

For any point $(x, y)$ in the plane except for the origin, let $\operatorname{atan}_{2}(y, x)$ be the angle between the horizontal positive axis and the point $(x, y)$ measured counterclockwise.

Assume $a, b$ and $c$ are constants in $\mathbb{R}$, consider the equation

$$
a \cos \theta+b \sin \theta=c,
$$

in the variable $\theta \in[-\pi, \pi[$. The following statements hold:

(i) if $a^{2}+b^{2}<c^{2}$, then equation (4) admits no solution;

(ii) if $a^{2}+b^{2}=c^{2}$, then equation (4) admits the unique solution

$$
\theta=\operatorname{atan}_{2}(b, a)+\operatorname{atan}_{2}(0, c) ;
$$

(iii) if $a^{2}+b^{2}>c^{2}$, then equation (4) admits the two solutions given by

$$
\theta_{1,2}=\operatorname{atan}_{2}(b, a) \pm \operatorname{atan}_{2}\left(\sqrt{a^{2}+b^{2}-c^{2}}, c\right) .
$$

In equations (5) and (6), the equality sign is meant modulo $2 \pi$ so that the solutions take value in $[-\pi, \pi[$. 


\section{BASIC INVERSION AND EQUALITY PROBLEMS INVOLVING ROTATIONS}

In this section we present four basic lemmas that have intrinsic interest and that are required by the proofs of the main results in the next section. The first two lemmas provide the complete solution to two decomposition problems for rotation matrices.

Lemma $1(\mathbf{S O}(3)$ inversion) Assume $\boldsymbol{v}$ and $\boldsymbol{w}$ are unit-length vectors in $\mathbb{R}^{3}$ and consider the equation

$$
v=R w
$$

in the variable $\boldsymbol{R} \in S O(3)$.

Every solution to equation (7) is written as

$$
\boldsymbol{R}=\exp (\beta \widehat{\boldsymbol{v}}) \exp (\alpha \widehat{\boldsymbol{e}})
$$

where $\beta$ is an arbitrary angle in $[-\pi, \pi[$, and where the angle $\alpha \in$ $[0, \pi]$ and the unit-length vector $e \in \mathbb{R}^{3}$ are defined by

$$
\begin{aligned}
\alpha & =\operatorname{atan}_{2}\left(\|v \times w\|, v^{T} w\right), \\
\boldsymbol{e} & = \begin{cases}\operatorname{vers}(v \times w), & \text { if } v \times w \neq \mathbf{0} \\
\text { any unit-length vector } \perp v, & \text { otherwise. }\end{cases}
\end{aligned}
$$

Proof: First, let us show that $\boldsymbol{H}=\exp (\alpha \widehat{\boldsymbol{e}})$ is a solution of (7):

$$
\begin{aligned}
\boldsymbol{H} \boldsymbol{w} & =\exp (\alpha \widehat{\boldsymbol{e}}) \boldsymbol{w}, \\
& =\boldsymbol{w} \cos \alpha+(\boldsymbol{e} \times \boldsymbol{w}) \sin \alpha+(1-\cos \alpha)(\boldsymbol{e} \cdot \boldsymbol{w}) \boldsymbol{w}
\end{aligned}
$$

Because $\boldsymbol{e}$ and $\boldsymbol{w}$ are mutually orthogonal unit vectors, we have that

$$
\boldsymbol{H} \boldsymbol{w}=\boldsymbol{w} \cos \alpha+\boldsymbol{n} \sin \alpha,
$$

where $\boldsymbol{n}$ is a unit vector perpendicular to the plane containing $\boldsymbol{e}$ and $\boldsymbol{w}$ whose direction is given by their cross product. Let us consider the orthonormal base $\{\boldsymbol{w}, \boldsymbol{n}, \boldsymbol{e}\}$. Then, equation (8) represents the rotation of axis $\boldsymbol{w}$ around axis $e$ of an angle $\alpha$, where $\alpha$ is, by definition, the angle between $\boldsymbol{w}$ and $\boldsymbol{v}$. Therefore $\boldsymbol{H} \boldsymbol{w}=\boldsymbol{v}$, that is, $\boldsymbol{H}$ is solution of (7). Now, for an arbitrary angle $\gamma \in[-\pi, \pi[$, we compute

$$
\begin{aligned}
\exp (\gamma \widehat{\boldsymbol{v}}) \boldsymbol{v} & =\boldsymbol{v} \cos \gamma+(\boldsymbol{v} \times \boldsymbol{v}) \sin \gamma+(1-\cos \gamma)(\boldsymbol{v} \cdot \boldsymbol{v}) \boldsymbol{v} \\
& =\boldsymbol{v} \cos \gamma+\boldsymbol{v}-\boldsymbol{v} \cos \gamma=\boldsymbol{v}
\end{aligned}
$$

Then $\boldsymbol{v}=\exp (\gamma \widehat{\boldsymbol{v}}) \boldsymbol{H} \boldsymbol{w}$.

Now, we want to show that any solution of (7) takes such a form. Suppose the matrix $\tilde{\boldsymbol{R}} \in S O(3)$ is solution of (7). We obtain $\tilde{\boldsymbol{R}} \boldsymbol{w}=\exp (\gamma \widehat{\boldsymbol{v}}) \boldsymbol{H} \boldsymbol{w}$, which can be easily written as $\exp (\alpha \widehat{\boldsymbol{e}}) \exp (\gamma \widehat{\boldsymbol{v}}) \tilde{\boldsymbol{R}} \boldsymbol{w}=\boldsymbol{w}$. It is known that any rotation of a fixed vector that yields the same vector is equivalent to a rotation of the vector about itself by any angle. Then

$$
\exp (\alpha \widehat{\boldsymbol{e}}) \exp (\gamma \widehat{\boldsymbol{v}}) \tilde{\boldsymbol{R}}=\exp (\delta \widehat{\boldsymbol{w}}),
$$

for any $\delta \in[-\pi, \pi[$. From (7) and (3) we obtain

$$
\exp (\delta \widehat{\boldsymbol{w}})=\exp \left(\delta \widehat{\tilde{\boldsymbol{R}}^{-1} \boldsymbol{v}}\right)=\tilde{\boldsymbol{R}}^{-1} \exp (\delta \widehat{\boldsymbol{w}}) \tilde{\boldsymbol{R}},
$$

and $\tilde{\boldsymbol{R}} \exp (\alpha \widehat{\boldsymbol{e}})=\exp ((\delta-\gamma) \widehat{\boldsymbol{v}})$. Therefore, any solution of (7) can be written as $\tilde{\boldsymbol{R}}=\exp (\varphi \widehat{\boldsymbol{v}}) \boldsymbol{H}$, for any $\varphi \in[-\pi, \pi[$.

Lemma $2(\operatorname{SO}(3)$ inversion about a fixed axis) Given unit-length vectors $\boldsymbol{v}, \boldsymbol{w}$, and $\boldsymbol{n}$ in $\mathbb{R}^{3}$ such that either $\boldsymbol{n}$ is not parallel to $\boldsymbol{v}$ or $\boldsymbol{n}$ is not parallel to $\boldsymbol{w}$. Then the following two statements are equivalent:

(i) $\boldsymbol{n}^{T} \boldsymbol{w}=\boldsymbol{n}^{T} \boldsymbol{v}$, and

(ii) there exists a unique angle $\theta \in[-\pi, \pi[$ such that

$$
\exp (\theta \widehat{\boldsymbol{n}}) \boldsymbol{v}=\boldsymbol{w}
$$

Furthermore, if the two statements hold, then

$$
\theta=\operatorname{atan}_{2}\left(\boldsymbol{w}^{T} \boldsymbol{n} \times \boldsymbol{v}, \boldsymbol{v}^{T} \boldsymbol{w}-\left(\boldsymbol{v}^{T} \boldsymbol{n}\right)\left(\boldsymbol{w}^{T} \boldsymbol{n}\right)\right) .
$$

Proof: To show that (ii) implies (i) it suffices to recall equation (2). To show that (i) implies (ii), note that both vectors $\boldsymbol{v}$ and $\boldsymbol{w}$ take value in the plane $\mathcal{H}$ perpendicular to $\boldsymbol{n}$ and passing through the points $\left(\boldsymbol{n}^{T} \boldsymbol{w}\right) \boldsymbol{n}$ and $\left(\boldsymbol{n}^{T} \boldsymbol{v}\right) \boldsymbol{n}$. Also note that $\boldsymbol{n}^{T} \boldsymbol{w}=\boldsymbol{n}^{T} \boldsymbol{v}$ is different from \pm 1 because of the assumption that $\boldsymbol{n}$ is not parallel to $\boldsymbol{v}$ or $\boldsymbol{w}$. We claim that (1) the intersection between the unit sphere $\mathbb{S}^{2}$ and the plane $\mathcal{H}$ is a circle and (2) the map $\theta \mapsto \exp (\theta \widehat{\boldsymbol{n}}) v$ is a bijection between $\mathbb{S}^{1}$ and the circle $\mathbb{S}^{2} \cap \mathcal{H}$. These claims follow from simple geometric arguments; see Figure 1. Because this map is

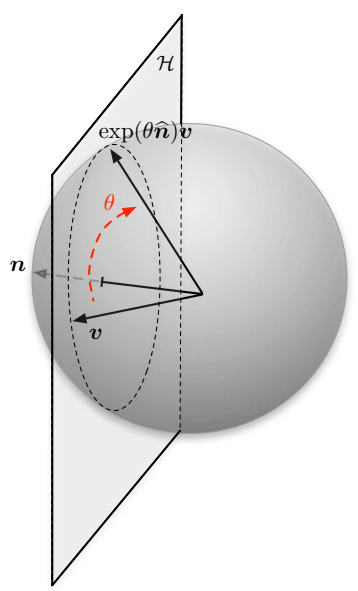

Fig. 1. A graphical proof of Lemma 2: there exists a unique angle $\theta \in$ $\left[-\pi, \pi\left[\right.\right.$ such that $\exp (\theta \widehat{\boldsymbol{n}}) \boldsymbol{v}=\boldsymbol{w}$ if and only if $\boldsymbol{n}^{T} \boldsymbol{w}=\boldsymbol{n}^{T} \boldsymbol{v}$.

bijective, we conclude that the angle $\theta$ is unique.

Finally, we prove the expression for the angle $\theta$. Let $\boldsymbol{v}_{\mathcal{H}}$ and $\boldsymbol{w}_{\mathcal{H}}$ be the orthogonal projections of $\boldsymbol{v}$ and $\boldsymbol{w}$ respectively onto the plane $\mathcal{H}$. Clearly, $\boldsymbol{v}_{\mathcal{H}}=\boldsymbol{v}-(\boldsymbol{v} \cdot \boldsymbol{n}) \boldsymbol{n}$. Geometric reasoning implies that $\theta$ is the amount of counter-clockwise rotation about the axis $\boldsymbol{n}$ that rotates $\boldsymbol{v}_{\mathcal{H}}$ onto $\boldsymbol{w}_{\mathcal{H}}$. On the plane $\mathcal{H}$, consider the orthonormal basis given by $\boldsymbol{v}_{\mathcal{H}} /\left\|\boldsymbol{v}_{\mathcal{H}}\right\|$ and $\boldsymbol{n} \times \boldsymbol{v}_{\mathcal{H}} /\left\|\boldsymbol{v}_{\mathcal{H}}\right\|$. We compute the angle $\theta$ by noting it must satisfy

$$
\cos (\theta)=\frac{\boldsymbol{v}_{\mathcal{H}} \cdot \boldsymbol{w}_{\mathcal{H}}}{\left\|\boldsymbol{v}_{\mathcal{H}}\right\|\left\|\boldsymbol{w}_{\mathcal{H}}\right\|}, \quad \text { and } \quad \sin (\theta)=\frac{\boldsymbol{w}_{\mathcal{H}} \cdot \boldsymbol{n} \times \boldsymbol{v}_{\mathcal{H}}}{\left\|\boldsymbol{v}_{\mathcal{H}}\right\|\left\|\boldsymbol{w}_{\mathcal{H}}\right\|},
$$

and, therefore,

$$
\theta=\operatorname{atan}_{2}(\sin \theta, \cos \theta)=\operatorname{atan}\left(\boldsymbol{w}_{\mathcal{H}} \cdot \boldsymbol{n} \times \boldsymbol{v}_{\mathcal{H}}, \boldsymbol{v}_{\mathcal{H}} \cdot \boldsymbol{w}_{\mathcal{H}}\right) .
$$

Simple calculations showing that $\boldsymbol{w}_{\mathcal{H}} \cdot \boldsymbol{n} \times \boldsymbol{v}_{\mathcal{H}}=\boldsymbol{w} \cdot \boldsymbol{n} \times \boldsymbol{v}$ and $\boldsymbol{v}_{\mathcal{H}} \cdot \boldsymbol{w}_{\mathcal{H}}=\boldsymbol{v} \cdot \boldsymbol{w}-(\boldsymbol{v} \cdot \boldsymbol{n})(\boldsymbol{w} \cdot \boldsymbol{n})$.

Our third and last result for this section provides necessary and sufficient conditions for two rotation matrices to be equal.

Lemma 3 (Equal rotations) Let $\boldsymbol{v}$ and $\boldsymbol{w}$ be unit-length vectors in $\mathbb{R}^{3}$ and let $\boldsymbol{R}, \tilde{\boldsymbol{R}} \in S O(3)$ be rotation matrices. If $\boldsymbol{R} \boldsymbol{w} \neq \pm \boldsymbol{v}$ or $\tilde{\boldsymbol{R}} \boldsymbol{w} \neq \pm \boldsymbol{v}$, then the following statements are equivalent:

(i) $\boldsymbol{R}=\tilde{\boldsymbol{R}}$,

(ii) $\boldsymbol{v}^{T} \tilde{\boldsymbol{R}}=\boldsymbol{v}^{T} \boldsymbol{R}$ and $\tilde{\boldsymbol{R}} \boldsymbol{w}=\boldsymbol{R} \boldsymbol{w}$.

If $\boldsymbol{w} \neq \pm \boldsymbol{v}$, then the following statements are equivalent:

(iii) $\boldsymbol{R}=\tilde{\boldsymbol{R}}$,

(iv) $\boldsymbol{v}^{T} \tilde{\boldsymbol{R}}=\boldsymbol{v}^{T} \boldsymbol{R}$ and $\boldsymbol{w}^{T} \tilde{\boldsymbol{R}}=\boldsymbol{w}^{T} \boldsymbol{R}$.

Proof: It is trivial to show that (i) implies (ii) and that (iii) implies (iv). We want now to show that (ii) implies (i). Without loss 
of generality, assume $\boldsymbol{R}^{T} \boldsymbol{v} \neq \pm \boldsymbol{w}$. From (ii) we have $\boldsymbol{R} \tilde{\boldsymbol{R}}^{T} \boldsymbol{v}=\boldsymbol{v}$ and $\tilde{\boldsymbol{R}}^{T} \boldsymbol{R} \boldsymbol{w}=\boldsymbol{w}$. Therefore, Lemma 1 implies that there exist angles $\alpha$ and $\beta$ in $[-\pi, \pi[$ such that

$$
\begin{aligned}
\boldsymbol{R} \tilde{\boldsymbol{R}}^{T} & =\exp (\alpha \widehat{\boldsymbol{v}}), \\
\tilde{\boldsymbol{R}}^{T} \boldsymbol{R} & =\exp (\beta \widehat{\boldsymbol{w}}) .
\end{aligned}
$$

From equation (9), using equation (3), we obtain

$$
\tilde{\boldsymbol{R}}^{T} \boldsymbol{R}=\exp \left(\alpha \widehat{R^{T} \boldsymbol{v}}\right),
$$

and, from equation (10), we have that

$$
\exp \left(\alpha \widehat{\boldsymbol{R}^{T} \boldsymbol{v}}\right)=\exp (\beta \widehat{\boldsymbol{w}}) .
$$

Equation (12) is satisfied if either $\alpha=\beta=0$ or $\boldsymbol{R}^{T} \boldsymbol{v}$ and $\boldsymbol{w}$ are parallel. However, by assumption we know $\boldsymbol{R}^{T} \boldsymbol{v} \neq \pm \boldsymbol{w}$. Therefore, equation (12) is true only if $\alpha=\beta=0$. This implies $\tilde{\boldsymbol{R}}^{T} \boldsymbol{R}=I$, and therefore $\boldsymbol{R}=\tilde{\boldsymbol{R}}$.

Now, let us show that (iv) implies (iii). Equations (iv) implies $\boldsymbol{R} \tilde{\boldsymbol{R}}^{T} \boldsymbol{v}=\boldsymbol{v}$ and $\boldsymbol{R} \tilde{\boldsymbol{R}}^{T} \boldsymbol{w}=\boldsymbol{w}$. According to Lemma 1, there exist angles $\alpha, \beta$ in $[-\pi, \pi[$ such that

$$
\begin{aligned}
& \boldsymbol{R} \tilde{\boldsymbol{R}}^{T}=\exp (\alpha \widehat{\boldsymbol{v}}), \\
& \boldsymbol{R} \tilde{\boldsymbol{R}}^{T}=\exp (\beta \widehat{\boldsymbol{w}}) .
\end{aligned}
$$

The following equivalence

$$
\exp (\alpha \widehat{\boldsymbol{v}})=\exp (\beta \widehat{\boldsymbol{w}})
$$

is satisfied only if $\alpha=\beta=0$ or if $\boldsymbol{v} \| \boldsymbol{w}$. We know by assumption that $\boldsymbol{v}$ and $\boldsymbol{w}$ are not parallel. Therefore, $\alpha=\beta=0$, that is, $\boldsymbol{R} \tilde{\boldsymbol{R}}^{T}=$ $I_{3}$, which implies $\boldsymbol{R}=\tilde{\boldsymbol{R}}$.

\section{DECOMPOSITION OF ROTATIONS: EXISTENCE AND COMPUTATION OF NON-ORTHOGONAL EULER ANGLES}

This section contains the main results of this note. First, we establish necessary and sufficient conditions for the existence of Euler angles about arbitrary rotation axes. In other words, we answer the following question: when is it possible to decompose a rotation matrix as the product of three rotation matrices about given arbitrary axes?

Theorem 4 (Decomposition of rotations: Existence) Let $\boldsymbol{r}_{1}, \boldsymbol{r}_{2}$ and $\boldsymbol{r}_{3}$ be unit-length vectors in $\mathbb{R}^{3}$ such that $\boldsymbol{r}_{2}$ is neither parallel to $\boldsymbol{r}_{1}$ nor to $\boldsymbol{r}_{3}$. The rotation matrix $\boldsymbol{R} \in S O(3)$ admits (possibly nonunique) Euler angles $\left\{\theta_{1}, \theta_{2}, \theta_{3}\right\} \in\left[-\pi, \pi\left[^{3}\right.\right.$ about $\left\{\boldsymbol{r}_{1}, \boldsymbol{r}_{2}, \boldsymbol{r}_{3}\right\}$, that is,

$$
\exp \left(\theta_{1} \widehat{\boldsymbol{r}}_{1}\right) \exp \left(\theta_{2} \widehat{\boldsymbol{r}}_{2}\right) \exp \left(\theta_{3} \widehat{\boldsymbol{r}}_{3}\right)=\boldsymbol{R},
$$

if and only if

$$
\left|\boldsymbol{r}_{1}^{T}\left(\boldsymbol{R}-\boldsymbol{r}_{2} \boldsymbol{r}_{2}^{T}\right) \boldsymbol{r}_{3}\right| \leq \sqrt{1-\left(\boldsymbol{r}_{1}^{T} \boldsymbol{r}_{2}\right)^{2}} \sqrt{1-\left(\boldsymbol{r}_{3}^{T} \boldsymbol{r}_{2}\right)^{2}} .
$$

Proof: First, let us start our proof by computing the condition such that equation (15) admits solutions in $\theta_{2}$. Left-multiplying by $\boldsymbol{r}_{1}^{T}$ and right-multiplying by $\boldsymbol{r}_{3}$ both sides of equation (13), equation (2) implies

$$
\boldsymbol{r}_{1}^{T} \exp \left(\theta_{2} \widehat{\boldsymbol{r}}_{2}\right) \boldsymbol{r}_{3}=\boldsymbol{r}_{1}^{T} \boldsymbol{R} \boldsymbol{r}_{3} .
$$

Decompose $\boldsymbol{r}_{1}$ and $\boldsymbol{r}_{3}$ as the orthogonal sum of their components parallel and perpendicular to $\boldsymbol{r}_{2}$ :

$$
\boldsymbol{r}_{1}=\left(\boldsymbol{r}_{1} \cdot \boldsymbol{r}_{2}\right) \boldsymbol{r}_{2}+\boldsymbol{r}_{1}^{\perp}, \quad \boldsymbol{r}_{3}=\left(\boldsymbol{r}_{3} \cdot \boldsymbol{r}_{2}\right) \boldsymbol{r}_{2}+\boldsymbol{r}_{3}^{\perp} .
$$

Using this decomposition and equation (2) we compute

$$
\boldsymbol{r}_{1}^{T} \exp \left(\theta_{2} \widehat{\boldsymbol{r}}_{2}\right) \boldsymbol{r}_{3}=\left(\boldsymbol{r}_{1} \cdot \boldsymbol{r}_{2}\right)\left(\boldsymbol{r}_{3} \cdot \boldsymbol{r}_{2}\right)+\boldsymbol{r}_{1}^{\perp} \exp \left(\theta_{2} \widehat{\boldsymbol{r}}_{2}\right) \boldsymbol{r}_{3}^{\perp},
$$

so that the scalar function

$$
\theta_{2} \mapsto \boldsymbol{r}_{1}^{T} \exp \left(\theta_{2} \widehat{\boldsymbol{r}}_{2}\right) \boldsymbol{r}_{3}-\left(\boldsymbol{r}_{1} \cdot \boldsymbol{r}_{2}\right)\left(\boldsymbol{r}_{3} \cdot \boldsymbol{r}_{2}\right)
$$

takes any value between $-\left\|\boldsymbol{r}_{1}^{\perp}\right\|\left\|\boldsymbol{r}_{3}^{\perp}\right\|$ and $\left\|\boldsymbol{r}_{1}^{\perp}\right\|\left\|\boldsymbol{r}_{3}^{\perp}\right\|$. Note that $\left\|\boldsymbol{r}_{1}^{\perp}\right\|^{2}=1-\left(\boldsymbol{r}_{1} \cdot \boldsymbol{r}_{2}\right)^{2}$ and $\left\|\boldsymbol{r}_{3}^{\perp}\right\|^{2}=1-\left(\boldsymbol{r}_{2} \cdot \boldsymbol{r}_{2}\right)^{2}$. Hence, equation (15) admits a (possibly non-unique) solution $\theta_{2}$ if and only if

$\left|\boldsymbol{r}_{1}^{T} \boldsymbol{R} \boldsymbol{r}_{3}-\left(\boldsymbol{r}_{1} \cdot \boldsymbol{r}_{2}\right)\left(\boldsymbol{r}_{3} \cdot \boldsymbol{r}_{2}\right)\right| \leq \sqrt{1-\left(\boldsymbol{r}_{1} \cdot \boldsymbol{r}_{2}\right)^{2}} \sqrt{1-\left(\boldsymbol{r}_{3} \cdot \boldsymbol{r}_{2}\right)^{2}}$,

which can be written as (14).

Next, we aim to prove that equation (13) has a (possibly nonunique) solution in $\left\{\theta_{1}, \theta_{2}, \theta_{3}\right\}$ if and only if equation (15) has a (possibly non-unique) solution in $\theta_{2}$.

To show that $\boldsymbol{R}$ and $\exp \left(\theta_{1} \widehat{\boldsymbol{r}}_{1}\right) \exp \left(\theta_{2} \widehat{\boldsymbol{r}}_{2}\right) \exp \left(\theta_{3} \widehat{\boldsymbol{r}}_{3}\right)$ are equal we adopt the results in Lemma 3 with two vectors $\boldsymbol{v}$ and $\boldsymbol{w}$ equal to $\boldsymbol{r}_{1}$ and $\boldsymbol{r}_{3}$. First, let us assume that $\boldsymbol{R} \boldsymbol{r}_{3} \neq \pm \boldsymbol{r}_{1}$. In order to use the first result in Lemma 3, we first right-multiply by $\boldsymbol{r}_{3}$ and, second, we left-multiply equation (13) by $\boldsymbol{r}_{1}^{T}$ and take the transpose. In summary, we obtain:

$$
\begin{aligned}
& \exp \left(\theta_{1} \widehat{\boldsymbol{r}}_{1}\right) \exp \left(\theta_{2} \widehat{\boldsymbol{r}}_{2}\right) \boldsymbol{r}_{3}=R \boldsymbol{r}_{3}, \\
& \exp \left(-\theta_{3} \widehat{\boldsymbol{r}}_{3}\right) \exp \left(-\theta_{2} \widehat{\boldsymbol{r}}_{2}\right) \boldsymbol{r}_{1}=R^{T} \boldsymbol{r}_{1} .
\end{aligned}
$$

Now, we invoke Lemma 2 once for equation (17) and once for equation (18). First, for equation (17), there exists a unique angle $\theta_{1} \in\left[-\pi, \pi\left[\right.\right.$ such that the unit-length vector $\exp \left(\theta_{2} \widehat{\boldsymbol{r}}_{2}\right) \boldsymbol{r}_{3}$ is rotated onto $R \boldsymbol{r}_{3}$ about the rotation axis $\boldsymbol{r}_{1}$ if and only if

$$
\boldsymbol{r}_{1}^{T}\left(R \boldsymbol{r}_{3}\right)=\boldsymbol{r}_{1}^{T}\left(\exp \left(\theta_{2} \widehat{\boldsymbol{r}}_{2}\right) \boldsymbol{r}_{3}\right) .
$$

Second, for equation (18), there exists a unique angle $\theta_{3} \in[-\pi, \pi[$ such that the unit-length vector $\exp \left(-\theta_{2} \widehat{\boldsymbol{r}}_{2}\right) \boldsymbol{r}_{1}$ is rotated onto $R^{T} \boldsymbol{r}_{1}$ about the rotation axis $\boldsymbol{r}_{3}$ if and only if

$$
\boldsymbol{r}_{3}^{T}\left(R^{T} \boldsymbol{r}_{1}\right)=\boldsymbol{r}_{3}^{T}\left(\exp \left(-\theta_{2} \widehat{\boldsymbol{r}}_{2}\right) \boldsymbol{r}_{1}\right) .
$$

Clearly, both equations (19) and (20) are equivalent to equation (15) and, assuming the inequality (14), they both hold true whenever $\theta_{2}$ is selected to satisfy equation (15).

Now, let us consider the special case

$$
\boldsymbol{R} \boldsymbol{r}_{3}= \pm \boldsymbol{r}_{1} .
$$

To show that equation (13) holds true, we want to invoke the second result of Lemma 3 with $\boldsymbol{w}$ and $\boldsymbol{v}$ equal to $\boldsymbol{r}_{2}$ and $\boldsymbol{r}_{1}$. Using equality (21), equation (13) can be written as:

$$
\exp \left( \pm \theta_{1} \widehat{\boldsymbol{R}}_{3}\right) \exp \left(\theta_{2} \widehat{\boldsymbol{r}}_{2}\right) \exp \left(\theta_{3} \widehat{\boldsymbol{r}}_{3}\right)=\boldsymbol{R}
$$

which is equivalent to

$$
\exp \left(\theta_{2} \widehat{\boldsymbol{r}}_{2}\right) \exp \left(\theta_{3} \widehat{\boldsymbol{r}}_{3}\right)=\exp \left(\mp \theta_{1} \widehat{\boldsymbol{R r}}_{3}\right) \boldsymbol{R} .
$$

Using the property (3) to the right-hand side, we obtain:

$$
\exp \left(\theta_{2} \widehat{\boldsymbol{r}}_{2}\right) \exp \left(\theta_{3} \widehat{\boldsymbol{r}}_{3}\right)=\boldsymbol{R} \exp \left(\mp \theta_{1} \widehat{\boldsymbol{r}}_{3}\right),
$$

and, therefore,

$$
\exp \left(\theta_{2} \widehat{\boldsymbol{r}}_{2}\right) \exp \left(\left(\theta_{3} \pm \theta_{1}\right) \widehat{\boldsymbol{r}}_{3}\right)=\boldsymbol{R} .
$$

Following a similar procedure, equation (13) can also be written as:

$$
\exp \left(\left(\theta_{1} \pm \theta_{3}\right) \widehat{\boldsymbol{r}}_{1}\right) \exp \left(\theta_{2} \widehat{\boldsymbol{r}}_{2}\right)=\boldsymbol{R} \text {. }
$$

Now, we left-multiply equation (22) by $\boldsymbol{r}_{2}^{T}$ and take the transpose. We obtain:

$$
\exp \left(-\left(\theta_{3} \pm \theta_{1}\right) \widehat{\boldsymbol{r}}_{3}\right) \boldsymbol{r}_{2}=\boldsymbol{R}^{T} \boldsymbol{r}_{2} .
$$

Since $\boldsymbol{r}_{2}$ is neither parallel to $\boldsymbol{r}_{1}$ nor to $\boldsymbol{r}_{3}$, we can use Lemma 2 . According to Lemma 2, there exists a unique angle $\theta_{3} \pm \theta_{1}$ in $[-\pi, \pi[$ 
such that the unit-length vector $\boldsymbol{r}_{2}$ is rotated onto $\boldsymbol{R}^{T} \boldsymbol{r}_{2}$ about the rotation axis $\boldsymbol{r}_{3}$ if and only if

$$
\boldsymbol{r}_{3}^{T} \boldsymbol{r}_{2}=\boldsymbol{r}_{3}^{T} \boldsymbol{R}^{T} \boldsymbol{r}_{2}
$$

which, for the equality (21), is equivalent to

$$
\boldsymbol{r}_{3}^{T} \boldsymbol{r}_{2}= \pm \boldsymbol{r}_{1}^{T} \boldsymbol{r}_{2}
$$

Now, we left-multiply equation (23) by $\boldsymbol{r}_{1}^{T}$ and take the transpose, and using equality (21) we obtain:

$$
\exp \left(-\theta_{2} \widehat{\boldsymbol{r}}_{2}\right) \boldsymbol{r}_{1}= \pm \boldsymbol{r}_{3},
$$

For Lemma 2, there exists a unique angle $\theta_{2}$ in $[-\pi, \pi[$ such that the unit-length vector $\boldsymbol{r}_{1}$ is rotated onto $\pm \boldsymbol{r}_{3}$ about the rotation axis $\boldsymbol{r}_{2}$ if and only if $\pm \boldsymbol{r}_{2}^{T} \boldsymbol{r}_{3}=\boldsymbol{r}_{2}^{T} \boldsymbol{r}_{1}$, which is equivalent to (25). Now, we want to show that (15) and (25) are equivalent whenever equality (21) holds. For (21), equation (15) becomes equivalent to

$$
\boldsymbol{r}_{1}^{T} \exp \left(\theta_{2} \widehat{\boldsymbol{r}}_{2}\right) \boldsymbol{r}_{3}= \pm 1,
$$

which is verified if and only if

$$
\exp \left(\theta_{2} \widehat{\boldsymbol{r}}_{2}\right) \boldsymbol{r}_{3}= \pm \boldsymbol{r}_{1} \text {. }
$$

If we left-multiply equation (28) by $\boldsymbol{r}_{2}^{T}$ and take the transpose, we obtain (25). Therefore, equation (15) implies (25). Vice versa, one more application of Lemma 2 shows that equation (25) implies (28) (and, therefore, (15)). Hence, we know that equations (24) and (26) hold true whenever $\theta_{2}$ is selected to satisfy equation (15).

This statement concludes our proof that equation (13) is solvable in $\left\{\theta_{1}, \theta_{2}, \theta_{3}\right\}$ if and only if equation (15) is solvable in $\theta_{2}$ and $\boldsymbol{R} \boldsymbol{r}_{3} \neq \pm \boldsymbol{r}_{1}$; and that equation (13) is solvable in $\left\{\theta_{2}, \theta_{3} \pm \theta_{1}\right\}$ if and only if equation (15) is solvable in $\theta_{2}$ and $\boldsymbol{R} \boldsymbol{r}_{3}= \pm \boldsymbol{r}_{1}$.

Next, we provide explicit formulas for the computation of the Euler angles about arbitrary axes.

Theorem 5 (Decomposition of rotations: Computation) Let $\boldsymbol{r}_{1}$, $\boldsymbol{r}_{2}$ and $\boldsymbol{r}_{3}$ be unit-length vectors in $\mathbb{R}^{3}$ such that $\boldsymbol{r}_{2}$ is neither parallel to $\boldsymbol{r}_{1}$ nor to $\boldsymbol{r}_{3}$. Assume that $\boldsymbol{r}_{1}, \boldsymbol{r}_{2}$ and $\boldsymbol{r}_{3}$ and the rotation matrix $\boldsymbol{R}$ satisfy the necessary and sufficient condition (14). Then all triplets of Euler angles $\left\{\theta_{1}, \theta_{2}, \theta_{3}\right\}$ satisfying equation (13) are determined as follows.

First, the angle $\theta_{2}$ is one of the two (possibly coincident) solutions to:

$$
\left(\theta_{2}\right)_{1,2}=\operatorname{atan}_{2}(b, a) \pm \operatorname{atan}_{2}\left(\sqrt{a^{2}+b^{2}-c^{2}}, c\right),
$$

where $a=-\boldsymbol{r}_{1}^{T} \widehat{\boldsymbol{r}}_{2}^{2} \boldsymbol{r}_{3}, b=\boldsymbol{r}_{1}^{T} \widehat{\boldsymbol{r}}_{2} \boldsymbol{r}_{3}$, and $c=\boldsymbol{r}_{1}^{T}\left(\boldsymbol{R}-I_{3}-\widehat{\boldsymbol{r}}_{2}^{2}\right) \boldsymbol{r}_{3}$.

Second, if $\boldsymbol{R}^{T} \boldsymbol{r}_{1} \neq \pm \boldsymbol{r}_{3}$, then the angles $\theta_{1}$ and $\theta_{3}$ are uniquely determined by

$$
\begin{aligned}
& \theta_{1}=\operatorname{atan}_{2}\left(\boldsymbol{w}_{1}^{T} \boldsymbol{r}_{1} \times \boldsymbol{v}_{1}, \boldsymbol{v}_{1}^{T} \boldsymbol{w}_{1}-\left(\boldsymbol{v}_{1}^{T} \boldsymbol{r}_{1}\right)\left(\boldsymbol{w}_{1}^{T} \boldsymbol{r}_{1}\right)\right), \\
& \theta_{3}=-\operatorname{atan}_{2}\left(\boldsymbol{w}_{3}^{T} \boldsymbol{r}_{3} \times \boldsymbol{v}_{3}, \boldsymbol{v}_{3}^{T} \boldsymbol{w}_{3}-\left(\boldsymbol{v}_{3}^{T} \boldsymbol{r}_{3}\right)\left(\boldsymbol{w}_{3}^{T} \boldsymbol{r}_{3}\right)\right),
\end{aligned}
$$

where $\boldsymbol{v}_{1}=\exp \left(\theta_{2} \widehat{\boldsymbol{r}}_{2}\right) \boldsymbol{r}_{3}, \boldsymbol{w}_{1}=\boldsymbol{R} \boldsymbol{r}_{3}, \boldsymbol{v}_{3}=\exp \left(-\theta_{2} \widehat{\boldsymbol{r}}_{2}\right) \boldsymbol{r}_{1}$, and $\boldsymbol{w}_{3}=\boldsymbol{R}^{T} \boldsymbol{r}_{1}$

Third, if $\boldsymbol{R}^{T} \boldsymbol{r}_{1}=\boldsymbol{r}_{3}$ (or $\boldsymbol{R}^{T} \boldsymbol{r}_{1}=-\boldsymbol{r}_{3}$ ), then the angles $\theta_{1}$ and $\theta_{3}$ are not uniquely determined, but their sum $\theta_{3}+\theta_{1}$ (respectively, their difference $\theta_{3}-\theta_{1}$ ) equals

$$
-\operatorname{atan}_{2}\left(\boldsymbol{w}_{\mathbf{2}}^{T} \boldsymbol{r}_{3} \times \boldsymbol{r}_{2}, \boldsymbol{r}_{2}^{T} \boldsymbol{w}_{2}-\left(\boldsymbol{r}_{2}^{T} \boldsymbol{r}_{3}\right)\left(\boldsymbol{w}_{2}^{T} \boldsymbol{r}_{3}\right)\right),
$$

for $\boldsymbol{w}_{2}=\boldsymbol{R}^{T} \boldsymbol{r}_{2}$

Proof: Left-multiplying by $\boldsymbol{r}_{1}^{T}$ and right-multiplying by $\boldsymbol{r}_{3}$ both sides of equation (13), equation (2) implies

$$
\boldsymbol{r}_{1}^{T} \exp \left(\theta_{2} \widehat{\boldsymbol{r}}_{2}\right) \boldsymbol{r}_{3}=\boldsymbol{r}_{1}^{T} \boldsymbol{R} \boldsymbol{r}_{3}
$$

Applying Rodrigues' formula (1) to the previous equation, we see that $\theta_{2}$ alone must satisfy

$$
a \cos \theta_{2}+b \sin \theta_{2}=c,
$$

where $a, b$ and $c$ are defined as in the proposition statement. Equation (13) has solutions only if equation (31) does. This fact follows as a consequence of the equivalence between $|c| \leq \sqrt{a^{2}+b^{2}}$ and (14). To show that, we consider the equivalence $\widehat{\boldsymbol{r}}_{2}^{2}=\boldsymbol{r}_{2} \boldsymbol{r}_{2}^{T}-I_{3}$. It is then easy to show that $c=\boldsymbol{r}_{1}^{T}\left(\boldsymbol{R}-\boldsymbol{r}_{2} \boldsymbol{r}_{2}^{T}\right) \boldsymbol{r}_{3}$. The right-hand side of inequality (14) can be written as

$$
\begin{aligned}
\sqrt{1-\left(\boldsymbol{r}_{1}^{T} \boldsymbol{r}_{2}\right)^{2}} \sqrt{1-\left(\boldsymbol{r}_{3}^{T} \boldsymbol{r}_{2}\right)^{2}} & =\left\|\boldsymbol{r}_{1}^{\perp}\right\|\left\|\boldsymbol{r}_{3}^{\perp}\right\| \\
& =\max _{\theta_{2}}\left|\left(\boldsymbol{r}_{1}^{\perp}\right)^{T} \exp \left(\theta_{2} \widehat{\boldsymbol{r}}_{2}\right) \boldsymbol{r}_{3}^{\perp}\right|,
\end{aligned}
$$

where $\boldsymbol{r}_{1}^{\perp}$ and $\boldsymbol{r}_{3}^{\perp}$ are defined as in (16), i.e., they are the component of $\boldsymbol{r}_{1}$ and $\boldsymbol{r}_{3}$ respectively, that is orthogonal to $\boldsymbol{r}_{2}$. Using the decomposition in (1), the following equivalence holds:

$$
\begin{aligned}
\left(\boldsymbol{r}_{1}^{\perp}\right)^{T} \exp \left(\theta_{2} \widehat{\boldsymbol{r}}_{2}\right) \boldsymbol{r}_{3}^{\perp}=-\left(\boldsymbol{r}_{1}^{\perp}\right)^{T} \widehat{\boldsymbol{r}}_{2}^{2} \boldsymbol{r}_{3}^{\perp} \cos \theta_{2} \\
+\left(\boldsymbol{r}_{1}^{\perp}\right)^{T} \widehat{\boldsymbol{r}}_{2} \boldsymbol{r}_{3}^{\perp} \sin \theta_{2}+\left(\boldsymbol{r}_{1}^{\perp}\right)^{T} \boldsymbol{r}_{3}^{\perp}+\left(\boldsymbol{r}_{1}^{\perp}\right)^{T} \widehat{\boldsymbol{r}}_{2}^{2} \boldsymbol{r}_{3}^{\perp} .
\end{aligned}
$$

Since $\boldsymbol{r}_{1}^{\perp}$ and $\boldsymbol{r}_{2}^{\perp}$ are orthogonal to $\boldsymbol{r}_{2}$, it follows that

$$
\begin{aligned}
&\left(\boldsymbol{r}_{1}^{\perp}\right)^{T} \boldsymbol{r}_{3}^{\perp}+\left(\boldsymbol{r}_{1}^{\perp}\right)^{T} \widehat{\boldsymbol{r}}_{2}^{2} \boldsymbol{r}_{3}^{\perp}=0,=-\left(\boldsymbol{r}_{1}^{\perp}\right)^{T} \widehat{\boldsymbol{r}}_{2}^{2} \boldsymbol{r}_{3}^{\perp}=-\boldsymbol{r}_{1}^{T} \widehat{\boldsymbol{r}}_{2}^{2} \boldsymbol{r}_{3}=a, \\
&\left(\boldsymbol{r}_{1}^{\perp}\right)^{T} \widehat{\boldsymbol{r}}_{2} \boldsymbol{r}_{3}^{\perp}=\boldsymbol{r}_{1}^{\perp} \widehat{\boldsymbol{r}}_{2} \boldsymbol{r}_{3}=b .
\end{aligned}
$$

Hence,

$$
\begin{aligned}
\sqrt{1-\left(\boldsymbol{r}_{1}^{T} \boldsymbol{r}_{2}\right)^{2}} & \sqrt{1-\left(\boldsymbol{r}_{3}^{T} \boldsymbol{r}_{2}\right)^{2}} \\
& =\max _{\theta_{2}}\left|\left(\boldsymbol{r}_{1}^{\perp}\right)^{T} \exp \left(\theta_{2} \widehat{\boldsymbol{r}}_{2}\right) \boldsymbol{r}_{3}^{\perp}\right| \\
& =\max _{\theta_{2}}\left|a \cos \theta_{2}+b \sin \theta_{2}\right|=\sqrt{a^{2}+b^{2}},
\end{aligned}
$$

and therefore the inequalities $|c| \leq \sqrt{a^{2}+b^{2}}$ and (14) are equivalent.

We just showed that equation (15) admits solutions if and only if equation (31) has solutions. Now, as a direct consequence of the basic trigonometric inversion in Subsection I-B we establish the following facts. If $c^{2}>a^{2}+b^{2}$, then equations (31) and (13) have no solutions. If $c^{2} \leq a^{2}+b^{2}$, then equation (15) has two real solutions, $\left(\theta_{2}\right)_{1}$ and $\left(\theta_{2}\right)_{2}$, as given in equation (29) in the statement, and these two solutions are identical only if $c^{2}=a^{2}+b^{2}$. The expressions for $\theta_{1}$ and $\theta_{3}$ as given in the statement follow directly from Lemma 2 in order to satisfy equations (17) and (18) respectively. The expression for $\theta_{3} \pm \theta_{1}$ in the statement follows from Lemma 2 applied to equation (24).

Finally, for completeness sake, we consider the special case of the decomposition of a rotation matrix as the product of two rotation matrices about given arbitrary axes.

Lemma 6 (Decomposition into two rotation matrices) Let $\boldsymbol{r}_{4}$ and $\boldsymbol{r}_{5}$ be unit-length vectors in $\mathbb{R}^{3}$ such that $\boldsymbol{r}_{4} \neq \pm \boldsymbol{r}_{5}$, and let $\boldsymbol{R}$ be a rotation matrix in $S O(3)$. The following equation

$$
\exp \left(\theta_{4} \widehat{\boldsymbol{r}}_{4}\right) \exp \left(\theta_{5} \widehat{\boldsymbol{r}}_{5}\right)=\boldsymbol{R},
$$

admits a unique solution $\left\{\theta_{4}, \theta_{5}\right\} \in\left[-\pi, \pi\left[^{2}\right.\right.$ if and only if

$$
\boldsymbol{r}_{4}^{T} \boldsymbol{r}_{5}=\boldsymbol{r}_{4}^{T} \boldsymbol{R} \boldsymbol{r}_{5} \text {. }
$$

Such solution is given by

$$
\begin{aligned}
& \theta_{4}=\operatorname{atan}_{2}\left(\boldsymbol{w}_{4}^{T} \boldsymbol{r}_{4} \times \boldsymbol{r}_{5}, \boldsymbol{r}_{5}^{T} \boldsymbol{w}_{4}-\left(\boldsymbol{r}_{5}^{T} \boldsymbol{r}_{4}\right)\left(\boldsymbol{w}_{4}^{T} \boldsymbol{r}_{4}\right)\right), \\
& \theta_{5}=-\operatorname{atan}_{2}\left(\boldsymbol{w}_{5}^{T} \boldsymbol{r}_{5} \times \boldsymbol{r}_{4}, \boldsymbol{r}_{4}^{T} \boldsymbol{w}_{5}-\left(\boldsymbol{r}_{4}^{T} \boldsymbol{r}_{5}\right)\left(\boldsymbol{w}_{5}^{T} \boldsymbol{r}_{5}\right)\right),
\end{aligned}
$$

where $\boldsymbol{w}_{4}=\boldsymbol{R} \boldsymbol{r}_{5}$ and $\boldsymbol{w}_{5}=\boldsymbol{R}^{T} \boldsymbol{r}_{4}$. 
Proof: It is straightforward to show that (32) implies (33). Let us now assume that (33) holds. For to Lemma 2, there exists a unique angle $\alpha$ such that

$$
\exp \left(\alpha \widehat{\boldsymbol{r}}_{4}\right) \boldsymbol{r}_{5}=\boldsymbol{R} \boldsymbol{r}_{5},
$$

which is equivalent to right-multiplying both sides of equation (32) by $\boldsymbol{r}_{5}$. Therefore, $\theta_{4}=\alpha$. Now, we take the transpose of equation (33). According to Lemma 2, there exists a unique angle $\beta$ such that

$$
\exp \left(\beta \widehat{\boldsymbol{r}}_{5}\right) \boldsymbol{r}_{4}=\boldsymbol{R}^{T} \boldsymbol{r}_{4},
$$

which is equivalent to left-multiplying both sides of equation (32) by $\boldsymbol{r}_{4}^{T}$ and take the transpose. Therefore, $\theta_{5}=-\beta$. Hence, for Lemma 3 , equation (32) holds.

The values for $\theta_{4}$ and $\theta_{5}$ follow from equations (34) and (35) when Lemma 2 is applied.

\section{EQUiVAlENCE WITH THE ClASSIC TREATMENT OF EULER ANGLES}

In this section we verify that our general conditions and formulas are consistent with the classic results. Specifically, we show how the results in Theorem 4 confirm the known existence results for the classic Euler angles, i.e., for the case in which the second rotation axis is orthogonal to both the first and the third rotation axes.

Corollary 7 (Existence for orthogonal axes) Adopting the same notation as in Theorem 4, if $\boldsymbol{r}_{1} \perp \boldsymbol{r}_{2}$ and $\boldsymbol{r}_{2} \perp \boldsymbol{r}_{3}$, then the necessary and sufficient condition (14) holds true always and therefore Euler angles are well defined for any $\boldsymbol{R} \in S O(3)$.

Proof: If $\boldsymbol{r}_{1} \perp \boldsymbol{r}_{2}$ and $\boldsymbol{r}_{2} \perp \boldsymbol{r}_{3}$, then $\boldsymbol{r}_{1}^{T} \boldsymbol{r}_{2}=0$ and $\boldsymbol{r}_{3}^{T} \boldsymbol{r}_{2}=0$. Therefore, equation (14) becomes

$$
\boldsymbol{r}_{1}^{T} \boldsymbol{R} \boldsymbol{r}_{3} \leq 1
$$

Since $\boldsymbol{r}_{1}$ and $\boldsymbol{r}_{3}$ are unit-length vectors, the above inequality is always satisfied. Hence, according to Theorem 4, Euler angles are always well defined for any $\boldsymbol{R} \in S O(3)$.

Finally, we verify the following fact: when our general formulas are applied to specific choices of mutually orthogonal axes, they are equivalent to the ones reported in the literature. Specifically, if $\boldsymbol{r}_{1}$ and $\boldsymbol{r}_{3}$ are equal to $\boldsymbol{z}$ and $\boldsymbol{r}_{2}$ is equal to $\boldsymbol{y}$, then equation (13) becomes

$$
\exp \left(\theta_{1} \widehat{\boldsymbol{z}}\right) \exp \left(\theta_{2} \widehat{\boldsymbol{y}}\right) \exp \left(\theta_{3} \widehat{\boldsymbol{z}}\right)=\boldsymbol{R}_{Z Y Z}
$$

and the rotation matrix $\boldsymbol{R}_{Z Y Z}=\left[R_{i j}\right], i \in\{1,2,3\}$, is called the ZYZ-Euler angle transformation. It is well known (see, for example, [1]) that solutions of equation (36) are given by

$$
\begin{gathered}
\theta_{2}=\operatorname{atan}_{2}\left( \pm \sqrt{1-R_{33}^{2}}, R_{33}\right), \\
\theta_{1}= \begin{cases}\operatorname{atan}_{2}\left(R_{23}, R_{13}\right), & \text { if } \sin \theta_{2}>0, \\
\operatorname{atan}_{2}\left(-R_{23},-R_{13}\right), & \text { if } \sin \theta_{2}<0,\end{cases}
\end{gathered}
$$

and

$$
\theta_{3}= \begin{cases}\operatorname{atan}_{2}\left(R_{32},-R_{31}\right), & \text { if } \sin \theta_{2}>0, \\ \operatorname{atan}_{2}\left(-R_{32}, R_{31}\right), & \text { if } \sin \theta_{2}<0 .\end{cases}
$$

If $\sin \theta_{2}=0$, and, consequently, $\cos \theta_{2}= \pm 1$, only the sum $\theta_{1} \pm \theta_{3}$ can be determined, and it is given by:

$$
\theta_{1} \pm \theta_{3}= \pm \operatorname{atan}_{2}\left(R_{21}, R_{22}\right) .
$$

Corollary 8 (ZYZ-Euler angle transformation) The Euler angles solutions of equation (36) can be equivalently computed by using the formulas provided by Theorem 5 applied to the special case $\boldsymbol{r}_{1}=$ $\boldsymbol{r}_{3}=\boldsymbol{z}$ and $\boldsymbol{r}_{2}=\boldsymbol{y}$.
Proof: Since $\boldsymbol{r}_{1}$ and $\boldsymbol{r}_{2}$ are equal to $\boldsymbol{z}$ and $\boldsymbol{r}_{2}$ is equal to $\boldsymbol{y}$, the parameters $a, b$ and $c$ in (29) become $a=1, b=0$ and $c=R_{33}$. Therefore, equation (29) becomes:

$$
\left(\theta_{2}\right)_{1,2}=\operatorname{atan}_{2}\left( \pm \sqrt{1-R_{33}^{2}}, R_{33}\right),
$$

which is equivalent to (37).

Now, let us consider the case $\boldsymbol{R}^{T} \boldsymbol{r}_{1} \neq \boldsymbol{r}_{3}$. According to Theorem 5 and substituting $\boldsymbol{r}_{1}$ and $\boldsymbol{r}_{3}$ with $\boldsymbol{z}$, and $\boldsymbol{r}_{3}$ with $\boldsymbol{y}$, angle $\theta_{1}$ is computed as $\theta_{1}=\operatorname{atan}_{2}\left(R_{23} \sin \theta_{2}, R_{13} \sin \theta_{2}\right)$. In particular, $\theta_{1}=$ $\operatorname{atan}_{2}\left(R_{23}, R_{13}\right)$ if $\sin \theta_{2}>0$, and $\theta_{1}=\operatorname{atan}_{2}\left(-R_{23},-R_{13}\right)$ if $\sin \theta_{2}<0$, as in (38). Following the same procedure, we can compute $\theta_{3}$, which, for Theorem 5 is $\theta_{3}=\operatorname{atan}_{2}\left(R_{32} \sin \theta_{2},-R_{31} \sin \theta_{2}\right)$. In particular, $\theta_{3}=\operatorname{atan}_{2}\left(R_{32},-R_{31}\right)$ if $\sin \theta_{2}>0$, and $\theta_{3}=$ $\operatorname{atan}_{2}\left(-R_{32}, R_{31}\right)$ if $\sin \theta_{2}<0$, as in (39).

Now, let us consider the case $\boldsymbol{R}^{T} \boldsymbol{r}_{1}= \pm \boldsymbol{r}_{3}$, which can be written as $\boldsymbol{R}^{T} \boldsymbol{z}= \pm \boldsymbol{z}$. From equation (29), it follows that $\theta_{2}=0$ if $\boldsymbol{R}^{T} \boldsymbol{z}=$ $\boldsymbol{z}$, and $\theta_{2}= \pm \pi$ if $\boldsymbol{R}^{T} \boldsymbol{z}=-\boldsymbol{z}$. Hence, the case $\boldsymbol{R}^{T} \boldsymbol{z}= \pm \boldsymbol{z}$ corresponds to $\sin \theta_{2}=0$ and $\cos \theta_{2}= \pm 1$. Then, from Theorem 5, the sum $\theta_{3} \pm \theta_{1}$ is given by $\theta_{3} \pm \theta_{1}=-\operatorname{atan}_{2}\left(-R_{21}, R_{22}\right)$, which is equivalent to (40). This concludes our proof that our results match the ZYZ-Euler angle transformation.

Other conventions for rotation axes and Euler angles triplets can be treated analogously.

\section{REFERENCES}

[1] M. W. Spong, S. Hutchinson, and M. Vidyasagar, Robot Modeling and Control. Wiley, 3 ed., 2006.

[2] J. J. Craig, Introduction to Robotics: Mechanics and Control. Prentice Hall, 3 ed., 2003.

[3] L. Sciavicco and B. Siciliano, Modeling and Control of Robot Manipulators. Springer, 2 ed., 2000.

[4] H. Goldstein, Classical Mechanics. Addison-Wesley, 2 ed., 1980.

[5] J. E. Marsden and T. S. Ratiu, Introduction to Mechanics and Symmetry. Springer, 2 ed., 1999.

[6] F. Bullo and A. D. Lewis, Geometric Control of Mechanical Systems. Texts in Applied Mathematics, Springer, 2004.

[7] S. Helgason, Differential Geometry, Lie Groups and Symmetric Spaces. Academic Press, 1978.

[8] R. M. Murray, Z. X. Li, and S. S. Sastry, A Mathematical Introduction to Robotic Manipulation. CRC Press, 1994.

[9] R. W. Brockett, "Some mathematical aspects of robotics," in Proceedings of Symposia in Applied Mathematics (R. W. Brockett, ed.), vol. 41, pp. 16-40, American Mathematical Society, 1990.

[10] M. T. Mason, Mechanics of Robotic Manipulation. MIT Press, 2001.

[11] B. Etkin and L. D. Reid, Dynamics of Flight: Stability and Control. Wiley, 1995.

[12] Y. Ma, S. Soatto, J. Košecká, and S. S. Sastry, An Invitation to 3-D Vision: From Images to Geometric Models. Springer, 2003.

[13] P. B. Davenport, "Rotations about nonorthogonal axes," AIAA Journal, vol. 11, no. 6, pp. 853-857, 1973.

[14] J. Wittenburg and L. Lilov, "Decomposition of a finite rotation into three rotations about given axes," Multibody System Dynamics, vol. 9, no. 4, pp. 353-375, 2003.

[15] M. D. Shuster and F. L. Markley, "Generalization of the Euler angles," Journal of the Astronautical Sciences, vol. 51, no. 2, pp. 123-132, 2003.

[16] S. Martínez, J. Cortés, and F. Bullo, "A catalog of inverse-kinematics planners for underactuated systems on matrix groups," Journal of Geometric Mechanics, vol. 1, no. 4, pp. 445-460, 2009.

[17] M. D. Shuster, "Deterministic three-axis attitude determination," The Journal of the Astronautical Sciences, vol. 52, no. 3, pp. 405-419, 2004.

[18] G. Piovan, I. Shames, B. Fidan, F. Bullo, and B. D. O. Anderson, "On frame and orientation localization for relative sensing networks," in IEEE Conf. on Decision and Control, (Cancún, México), pp. 2326-2331, Dec. 2008. Journal version conditionally accepted in Automatica. 